\title{
Severe maternal morbidity: admission shift from intensive care unit to obstetric high- dependency unit
}

\author{
Ning Gu, Yaning Zheng and Yimin Dai
}

\begin{abstract}
Background: To study temporal trends of intensive care unit (ICU) admission in obstetric population after the introduction of obstetric high-dependency unit (HDU).

Methods: This is a retrospective study of consecutive obstetric patients admitted to the ICU/HDU in a provincial referral center in China from January 2014 to December 2019. The collected information included maternal demographic characteristics, indications for ICU and HDU admission, the length of ICU stay, the total length of in-hospital stay and APACHE II score. Chi-square and ANOVA tests were used to determine statistical significance. The temporal changes were assessed with chi-square test for linear trend.
\end{abstract}

Results: A total of 40,412 women delivered and 447 (1.11\%) women were admitted to ICU in this 6-year period. The rate of ICU admission peaked at 1.59\% in 2016 and then dropped to $0.67 \%$ in 2019 with the introduction of obstetric HDU. The average APACHE II score increased significantly from 6.8 to $12.3(\mathrm{P}<0.001)$ and the average length of ICU stay increased from 1.7 to 7.1 days $(P<0.001)$. The main indications for maternal ICU admissions were hypertensive disorders in pregnancy (39.8\%), cardiac diseases (24.8\%), and other medical disorders (21.5\%); while the most common reasons for referring to HDU were hypertensive disorders of pregnancy (46.5\%) and obstetric hemorrhage (43.0\%). The establishment of HDU led to 20\% reduction in ICU admission, which was mainly related to obstetric indications.

Conclusions: The introduction of HDU helps to reduce ICU utilization in obstetric population.

Keywords: Severe maternal morbidity, Intensive care unit, High dependency unit, Hypertensive disorders in pregnancy, Pre-existing medical disease

\section{Background}

Admission of pregnant or postpartum women to intensive care unit (ICU) is an indicator of severe maternal morbidity and an endpoint for clinical audit in quality of care [1]. Despite the proportion of high-risk pregnancies in China increased from 15.7 to $24.7 \%$, maternal mortality rates decreased from 34.2 per 100,000 to 18.3 per 100,000 live births from 2008 to 2018 [2], which implied

\footnotetext{
*Correspondence: nj_daiyimin@126.com
}

Department of Obstetrics and Gynecology, Nanjing University Medical School Affiliated Nanjing Drum Tower Hospital, 321 Zhongshan Road, Nanjing 210008, China an improvement in perinatal care. On the other hand, the growth of complicated pregnancies caused an increase in maternal ICU admissions [3], which meant a rising need of medical resources and expenses. Therefore, monitoring of the trend of ICU admission may help us understand the reasons for the change and develop interventions to improve medical service.

High dependency unit (HDU) provides a level of care which lies in between a general ward and an ICU, which has been suggested to reduce the need for ICU beds [4]. As previous researches from China showed, the incidence of pregnancy related ICU admission varied from 
0.56 to $1.6 \%[3,5]$. However, little was known about the respective roles of obstetric HDU and ICU in caring severe maternal morbidity in this region. Accordingly, the present study aims to study the temporal trend of ICU admission with the introduction of maternal HDU and assess the characteristics of pregnancy-related ICU and HDU admissions.

\section{Methods}

\section{Study design and settings}

This is a retrospective study of consecutive obstetric patients admitted to the ICU/HDU of Nanjing Drum Tower Hospital from January 2014 to December 2019. The obstetric service in this hospital has high patient acuity and was accredited as a provincial referral center for severe maternal morbidity in 2016. To cope with the rising demand for a higher level of care, the institute introduced obstetric HDU in 2017. The general ICU was a 20-bed unit led by intensivists, whereas the obstetric HDU was a 5-bed unit staffed with obstetricians and specialized nurses.

The research has been approved by the Ethical Committee of Nanjing Drum Tower Hospital and a waiver of individual informed consent was granted. Information of cases admitted to ICU of any causes during the pregnancy or within 42 days postpartum was abstracted from medical records and did not identify participants. Women delivered in other institutions and transferred to the hospital in postpartum period were excluded in the analysis.

\section{Participants and Variables}

The women's characteristics included age, parity, type of pregnancy (singleton or multiple), gestational age of delivery and mode of delivery (vaginal or cesarean delivery). The ICU admission information included the length of ICU stay, the total length of in-hospital stay and APACHE II score [6], calculated within 24h of ICU admission based on the worst clinical, and physiologic indicators. The indications for ICU/HDU admissions were classified into obstetric and non-obstetric complications. Organ dysfunction was defined using the WHO Working Group approach [7], which included clinical criteria, laboratory markers and management-based proxies. If a woman was admitted to the ICU more than once, the stay with the worst clinical manifestations (the highest APACHE II score or the longest stay) were included in this study.

\section{Statistical methods}

Statistical analysis was performed with SPSS 26.0. Normally distributed data were presented as mean with $\mathrm{SD}$; and categorical outcomes were summarized using frequency distributions. Chi-square and ANOVA tests were used to determine statistical significance. The temporal changes were assessed with chi-square test for linear trend.

To estimate the associations between maternal clinical features and ICU admission, characteristics (age, obesity, multiple pregnancy, pre-gestational hypertension, pregestational diabetes, pre-eclampsia, and placenta previa) for women with and without ICU admission were compared using Chi-square tests, odds ratios (OR) and 95\% confidence intervals (CI). Multiple logistic regression was used to estimate OR of ICU admission while adjusting for maternal risk factors and the introduction of HDU. A p value of 0.05 was used as the cut-point for significance.

\section{Results}

During this 6-year period, a total of 40,412 women delivered at the hospital and 447 women were admitted to ICU, making an overall ICU admission rate of $1.11 \%$. The rate peaked at $1.59 \%$ in 2016 and then dropped to $0.67 \%$ in 2019 (Table 1).

There were 9 maternal deaths and the maternal mortality rates were 2.0 per $100 \mathrm{ICU}$ admissions and 22.3 per 100,000 hospital deliveries. No significant differences were found regarding the yearly ICU mortality rates. Cardiac diseases were the leading cause of maternal death (5/9, including 3 cases of severe pulmonary hypertension, one case of heart failure secondary to Sjogren syndrome and one woman with prosthetic valve thrombosis), followed by infections $(2 / 9$, one with severe virus pneumonia and one with septic miscarriage), lymphoma (1/9) and cerebral hemorrhage (1/9).

\section{Demographics of Pregnancy-related ICU admission}

Overall, the mean age of the women admitted to the ICUs was 29.4 years; $16.8 \%$ were 35 years of age or older (Table 1). Nulliparous women comprised $65.1 \%$ of the ICU admissions, and the proportion decreased over time $(\mathrm{P}<0.001)$. Most of the patients were admitted during the postpartum period $(88.1 \%)$ and had undergone cesarean deliveries $(85.7 \%)$.

The average APACHE II score, an indicator of severity, increased significantly from 6.8 in 2014 to 12.3 in 2019 $(\mathrm{P}<0.001)$. At the same time, the average length of ICU stay increased from 1.7 day to 7.1 days $(\mathrm{P}<0.001)$, and the mean hospital stay increased from 8.4 to 14.1 days $(\mathrm{P}=0.002)$.

\section{Indications for maternal ICU admission}

The main causes for maternal ICU admission were hypertensive disorders in pregnancy $(39.8 \%)$, cardiac diseases (24.8\%), and other medical disorders (21.5\%) (Table 2). Women could have had more than one complication. 
Table 1 Characteristics of women with pregnancy-related ICU admissions

\begin{tabular}{|c|c|c|c|c|c|c|c|c|}
\hline & Overall & 2014 & 2015 & 2016 & 2017 & 2018 & 2019 & $\mathbf{P}$ \\
\hline ICU admission & $n=447$ & $\mathrm{n}=52$ & $\mathrm{n}=56$ & $n=117$ & $\mathrm{n}=102$ & $n=70$ & $\mathrm{n}=50$ & \\
\hline No. of deliveries & 40,412 & 5737 & 5269 & 7357 & 7428 & 7107 & 7514 & \\
\hline Rate of ICU admission (\%) & 1.11 & 0.91 & 1.06 & 1.59 & 1.37 & 0.98 & 0.67 & $<0.001$ \\
\hline Age $($ mean $\pm s d)$ & $29.4 \pm 5.3$ & $29.1 \pm 5.3$ & $29.1 \pm 5.7$ & $29.1 \pm 4.9$ & $29.6 \pm 4.8$ & $29.6 \pm 5.8$ & $30.3 \pm 5.7$ & 0.818 \\
\hline Age $\geq 35$ years $n(\%)$ & $75(16.8)$ & $7(13.5)$ & $12(21.4)$ & $17(14.5)$ & 17(16.7) & $11(15.7)$ & $11(22.0)$ & 0.545 \\
\hline Nulliparous n (\%) & $291(65.1)$ & $47(90.4)$ & $47(83.9)$ & $71(60.7)$ & $59(57.8)$ & $37(52.9)$ & $30(60.0)$ & $<0.001$ \\
\hline Multiple pregnancy n (\%) & $39(8.7)$ & $5(9.6)$ & $2(3.6)$ & $12(10.3)$ & 11(10.8) & $5(7.1)$ & $4(8.1)$ & 0.691 \\
\hline Pre-gestational $\mathrm{BMI} \geq 28 \mathrm{~kg} / \mathrm{m}^{2} \mathrm{n}(\%)$ & $25(5.6)$ & $2(3.8)$ & $5(8.9)$ & $3(2.6)$ & $4(3.9)$ & $6(8.6)$ & $5(10.0)$ & 0.209 \\
\hline GA at delivery ${ }^{\mathrm{a}}$ & & & & & & & & 0.145 \\
\hline$<28$ wks n (\%) & $63(14.2)$ & $5(9.6)$ & $14(25.0)$ & $12(10.3)$ & $13(12.7)$ & $8(11.6)$ & $11(22.0)$ & \\
\hline $28-31^{+6}$ wks n (\%) & $86(19.3)$ & $8(15.4)$ & $11(19.6)$ & $21(18.1)$ & $24(23.5)$ & $11(15.9)$ & $11(22.0)$ & \\
\hline $32-36^{+6}$ wks n (\%) & $174(39.1)$ & $17(32.7)$ & $16(28.6)$ & $51(44.0)$ & $40(39.2)$ & $32(46.4)$ & $18(36.0)$ & \\
\hline$\geq 37$ wks n (\%) & $122(27.4)$ & $22(42.3)$ & $15(26.8)$ & $32(27.6)$ & $25(24.5)$ & $18(26.1)$ & $10(20.4)$ & \\
\hline Cesarean deliveries $\mathrm{n}(\%)$ & $383(85.7)$ & $45(86.5)$ & $45(80.4)$ & $104(88.9)$ & $89(87.3)$ & $60(85.7)$ & $40(80.0)$ & 0.662 \\
\hline Postpartum admission n (\%) & $394(88.1)$ & $49(94.2)$ & $52(92.9)$ & $108(92.3)$ & $94(92.2)$ & $57(81.4)$ & $34(68.0)$ & $<0.001$ \\
\hline APACHE II score (mean \pm sd) & $10.5 \pm 5.6$ & $6.8 \pm 3.6$ & $9.4 \pm 8.1$ & $11.3 \pm 5.0$ & $10.9 \pm 4.8$ & $10.9 \pm 5.3$ & $12.3 \pm 5.2$ & $<0.001$ \\
\hline Length of ICU stay (d) (mean \pm sd) & $4.5 \pm 6.6$ & $1.7 \pm 1.6$ & $3.3 \pm 4.5$ & $3.7 \pm 4.2$ & $5.2 \pm 8.6$ & $5.9 \pm 7.4$ & $7.1 \pm 8.5$ & $<0.001$ \\
\hline In-hospital stay (d) (mean \pm sd) & $11.7 \pm 8.8$ & $8.4 \pm 3.6$ & $10.4 \pm 6.9$ & $10.8 \pm 6.9$ & $12.5 \pm 10.1$ & $13.8 \pm 11.5$ & $14.1 \pm 9.8$ & 0.002 \\
\hline ICU mortality rate $\mathrm{n}(\%)$ & $9(1.9)$ & $0(0)$ & $1(1.8)$ & $2(1.7)$ & $3(2.9)$ & $1(1.4)$ & $2(4.0)$ & 0.802 \\
\hline
\end{tabular}

${ }^{a}$ Two cases of maternal death before delivery in 2016 and 2018 respectively

Table 2 Indications for Pregnancy-Related ICU Admissions ${ }^{2}$

\begin{tabular}{|c|c|c|c|c|c|c|c|c|}
\hline & Overall & 2014 & 2015 & 2016 & 2017 & 2018 & 2019 & $P$ trend \\
\hline No. of ICU admission & 447 & 52 & 56 & 117 & 102 & 70 & 50 & \\
\hline \multicolumn{9}{|l|}{ Obstetric Indications } \\
\hline Hemorrhage & $56(12.5)$ & $13(25.0)$ & $3(5.4)$ & $18(15.4)$ & $13(12.7)$ & $7(10.0)$ & $2(4.0)$ & 0.015 \\
\hline Hypertensive disorders & $178(39.8)$ & $24(46.2)$ & $26(46.4)$ & $45(38.5)$ & $46(45.1)$ & $23(32.9)$ & $14(28.0)$ & 0.034 \\
\hline Obstetric infections & $11(2.5)$ & $1(1.9)$ & $3(5.4)$ & $2(1.7)$ & $2(2.0)$ & $3(4.3)$ & $0(0)$ & 0.609 \\
\hline Amniotic fluid embolism & $4(0.9)$ & $2(3.8)$ & $0(0)$ & $1(0.9)$ & $0(0)$ & $0(0)$ & $1(2.0)$ & 0.319 \\
\hline Acute fatty liver of pregnancy & $17(3.8)$ & $2(3.8)$ & $0(0)$ & $5(4.3)$ & $6(5.9)$ & $3(4.3)$ & $1(2.0)$ & 0.740 \\
\hline Other obstetric complications & $6(1.3)$ & $1(1.9)$ & $2(3.6)$ & $1(0.9)$ & $1(1.0)$ & $1(1.4)$ & $0(0)$ & 0.272 \\
\hline \multicolumn{9}{|l|}{ Non-obstetric Indications } \\
\hline Cardiac diseases & $111(24.8)$ & $14(26.9)$ & $12(21.4)$ & $20(17.1)$ & $23(22.5)$ & $21(30.0)$ & $21(42.0)$ & 0.024 \\
\hline Non-obstetric infections & $16(3.6)$ & $0(0)$ & $1(1.8)$ & $3(2.6)$ & $6(5.9)$ & $4(5.7)$ & $2(4.0)$ & 0.071 \\
\hline Pancreatitis & $26(5.8)$ & $0(0)$ & $2(3.6)$ & $7(6.0)$ & $7(6.9)$ & $5(7.1)$ & $5(10.0)$ & 0.024 \\
\hline Other medical disorders & $96(21.5)$ & $16(30.8)$ & $12(21.4)$ & $26(22.2)$ & $20(19.6)$ & $9(12.9)$ & $13(26.0)$ & 0.190 \\
\hline \multicolumn{9}{|l|}{ Organ Dysfunctions } \\
\hline Cardiovascular dysfunction & $83(18.6)$ & $3(5.8)$ & $5(8.9)$ & $14(12.0)$ & $23(22.5)$ & $22(31.4)$ & $16(32.0)$ & $<0.001$ \\
\hline Respiratory dysfunction & $130(29.1)$ & $6(11.5)$ & $16(28.6)$ & $40(34.2)$ & $24(23.5)$ & $22(31.4)$ & $22(44.0)$ & 0.007 \\
\hline Renal dysfunction & $40(8.9)$ & $0(0)$ & $7(12.5)$ & $8(6.8)$ & $15(14.7)$ & $5(7.1)$ & $5(10.0)$ & 0.170 \\
\hline Hematological dysfunction & $112(25.1)$ & $12(23.1)$ & $14(25.0)$ & $31(2.6)$ & $26(25.5)$ & $14(20.0)$ & $15(30.0)$ & 0.832 \\
\hline Hepatic dysfunction & $17(3.8)$ & $2(3.8)$ & $1(1.8)$ & $7(6.0)$ & $6(5.9)$ & $0(0)$ & $1(2.0)$ & 0.420 \\
\hline Neurological dysfunction & $20(4.5)$ & $0(0)$ & $3(5.4)$ & $6(5.1)$ & $5(4.9)$ & $1(1.4)$ & $5(10.0)$ & 0.182 \\
\hline Uterine dysfunction & $13(2.9)$ & $2(3.8)$ & $2(3.6)$ & $5(4.3)$ & $1(1.0)$ & $2(2.9)$ & $1(2.0)$ & 0.366 \\
\hline
\end{tabular}

a Patients can have more than one indication 
Over the 6-year period, the proportion of obstetric hemorrhage (from 25.0 to $4.0 \% ; \mathrm{P}=0.015$ ) and hypertensive disorders (from 46.2 to $28.0 \%$; $\mathrm{P}=0.034$ ) decreased significantly, whereas the percentage of cardiac diseases increased from 26.9 to $42.0 \%(\mathrm{P}=0.024)$.

In the analysis of the breakdown of maternal organ dysfunctions (Table 2), respiratory dysfunction (29.1\%) had the highest incidence, followed by hematological dysfunction (25.1\%) and cardiovascular dysfunction (18.6\%). From 2014 to 2019, both respiratory dysfunction rates $(11.5-44.0 \%, \mathrm{P}=0.007)$ and cardiovascular dysfunction rates $(5.8-32.0 \%, \mathrm{P}<0.001)$ showed increasing trends.

\section{Decrease in ICU admission after introduction of HDU}

After the introduction of HDU, the rate of ICU admission reduced by $18.0 \%$ (1.23\% in $2014-16$ vs. $1.01 \%$ in 2017 19), and HDU admission (1575 cases) amounted to $7.15 \%$ of total obstetric population. The obstetric indications for HDU admissions were hypertensive disorders of pregnancy (733, 46.5\%), obstetric hemorrhage (678, 43.0\%), other pregnancy complications (16, 1.0\%), and obstetric infections $(2,0.1 \%)$. Heart diseases $(74,4.7 \%)$, other medical disorders $(161,10.2 \%)$, pancreatitis $(6,0.4 \%)$, and non-obstetric infections $(3,0.2 \%)$ accounted for the non-obstetric causes. The cause-specific rates of pregnancy-related ICU admissions significantly decreased for hypertensive disorders $(\mathrm{OR}=0.573,95 \% \mathrm{CI}=0.424$ $0.775)$ and obstetric hemorrhage $(\mathrm{OR}=0.518,95 \% \mathrm{CI}$ $=0.302-0.888$ ) in 2017-2019 (Table 3).

\section{Contribution of maternal risk factors to ICU admission}

In the obstetric population, the incidence of maternal age $\geq 35$ years $(10.5-14.3 \%, \mathrm{P}<0.001)$, pre-gestational $\mathrm{BMI} \geq 28 \mathrm{~kg} / \mathrm{m}^{2}(0.4-3.5 \%, \mathrm{P}<0.001)$, multiple pregnancies $(3.9-5.0 \%, \mathrm{P}<0.001)$, pre-gestational hypertension $(1.2-1.8 \%, \mathrm{P}=0.001)$ and preeclampsia $(4.9-6.3 \%$, $\mathrm{P}<0.001)$ increased significantly from 2014 to 2019 (Table 4).

Associations between maternal risk factors and ICU admission are included in Table 5. Women with preeclampsia were found to have the highest adjusted odds of ICU admission ( $\mathrm{aOR}=7.752,95 \% \mathrm{CI}=6.211$ 9.709), followed by placenta previa ( $\mathrm{aOR}=3.571,95 \%$ $\mathrm{CI}=2.463-5.181$ ) and pre-gestational diabetes (aOR $=2.538,95 \% \mathrm{CI}=1.656-3.891$ ). After adjusting for the maternal risk factors, the establishment of HDU was related to $20 \%$ reduction of $\mathrm{ICU}$ admission $(\mathrm{aOR}=0.796$, 95\% CI $=0.658-0.962$ ). Pre-gestational obesity, multiple

Table 3 Indications for HDU and ICU admissions before and after establishment of HDU ${ }^{\mathrm{a}}$

\begin{tabular}{|c|c|c|c|c|c|c|c|}
\hline & \multicolumn{2}{|c|}{ 2014-2016 } & \multicolumn{4}{|c|}{ 2017-2019 } & \multirow[t]{2}{*}{ OR $(95 \% \mathrm{Cl})^{b}$} \\
\hline & $\mathbf{n}$ & ICU admission $\mathrm{n}(\%)$ & $\mathrm{n}$ & $\begin{array}{l}\text { ICU and HDU } \\
\text { admission } n(\%)\end{array}$ & HDU admission n (\%) & ICU admission n (\%) & \\
\hline Obstetric hemorrhage & 2539 & $34(1.3)$ & 3151 & $700(22.2)$ & $678(21.5)$ & $22(0.7)$ & $0.518(0.302-0.888)$ \\
\hline Hypertensive disorders & 1468 & $95(6.5)$ & 2176 & $816(37.5)$ & $733(33.7)$ & $83(3.8)$ & $0.573(0.424-0.775)$ \\
\hline Obstetric infections & 207 & $6(2.9)$ & 333 & $7(2.1)$ & $2(0.6)$ & $5(1.5)$ & $0.511(0.154-1.695)$ \\
\hline Cardiac diseases & 263 & $46(17.5)$ & 403 & $139(27.6)$ & $74(18.4)$ & $65(16.1)$ & $0.907(0.599-1.373)$ \\
\hline Non-obstetric infections & 25 & $4(16.0)$ & 37 & $15(40.5)$ & $3(8.1)$ & $12(32.4)$ & $2.520(0.707-8.988)$ \\
\hline Pancreatitis & 16 & $9(56.3)$ & 31 & $23(74.2)$ & $6(19.4)$ & $17(54.8)$ & $0.944(0.280-3.183)$ \\
\hline
\end{tabular}

${ }^{a}$ Patients can have more than one indication. ${ }^{b}$ Odds ratios for indication-specific ICU admission rates before and after establishment of HDU

Table 4 Temporal Trends in the proportion of women with high-risk pregnancies ${ }^{a}$

\begin{tabular}{|c|c|c|c|c|c|c|c|}
\hline & 2014 & 2015 & 2016 & 2017 & 2018 & 2019 & P trend \\
\hline No. of deliveries & 5737 & 5269 & 7357 & 7428 & 7107 & 7514 & \\
\hline Maternal age $\geq 35$ years & $603(10.5)$ & $741(14.1)$ & $1068(14.5)$ & $1387(18.7)$ & $1119(15.7)$ & $1071(14.3)$ & $<0.001$ \\
\hline Multiple pregnancies & $224(3.9)$ & $282(5.4)$ & $342(4.6)$ & $413(5.6)$ & $442(6.2)$ & $377(5.0)$ & $<0.001$ \\
\hline Pre-gestational $\mathrm{BMI} \geq 28 \mathrm{~kg} / \mathrm{m}^{2}$ & $24(0.4)$ & $110(2.1)$ & $140(1.9)$ & $134(1.8)$ & $187(2.6)$ & $265(3.5)$ & $<0.001$ \\
\hline Pre-gestational hypertension & $71(1.2)$ & $72(1.4)$ & $119(1.6)$ & $142(1.9)$ & $128(1.8)$ & $138(1.8)$ & 0.001 \\
\hline Pre-gestational diabetes & $92(1.6)$ & $62(1.2)$ & $76(1.0)$ & $71(1.0)$ & $90(1.3)$ & $117(1.6)$ & 0.768 \\
\hline Placenta previa & $115(2.0)$ & $155(2.9)$ & $176(2.4)$ & $211(2.8)$ & $193(2.7)$ & $172(2.3)$ & 0.519 \\
\hline Gestational diabetes & 1107 (19.3) & $527(10.0)$ & 779 (10.6) & $930(12.5)$ & $763(10.7)$ & $798(10.6)$ & $<0.001$ \\
\hline Preeclampsia & $280(4.9)$ & $279(5.3)$ & $444(6.0)$ & $468(6.3)$ & $437(6.1)$ & $475(6.3)$ & $<0.001$ \\
\hline
\end{tabular}

${ }^{a}$ Values are given as number (percentage) 
Table 5 Maternal risk factors for ICU admission

\begin{tabular}{lll}
\hline & Crude OR (95\% Cl) & Adjusted OR (95\% Cl) $^{\mathbf{a}}$ \\
\hline Maternal age $\geq 35$ years & $1.161(0.904-1.490)$ & - \\
Multiple birth & $1.776(1.275-2.474)$ & $1.211(0.860-1.706)$ \\
Pre-gestational BMI & $2.776(1.844-4.180)$ & $1.073(0.676-1.704)$ \\
$\geq 28 \mathrm{~kg} / \mathrm{m}^{2}$ & & \\
Pre-gestational hyperten- & $4.459(2.952-6.736)$ & $0.977(0.621-1.536)$ \\
sion & & \\
Pre-gestational diabetes & $4.731(3.154-7.049)$ & $2.538(1.656-3.891)$ \\
Placenta previa & $2.944(2.044-4.241)$ & $3.571(2.463-5.181)$ \\
Gestational diabetes & $0.790(0.578-1.081)$ & - \\
Preeclampsia & $8.002(6.540-9.791)$ & $7.752(6.211-9.709)$ \\
Establishment of HDU & $0.820(0.680-0.988)$ & $0.796(0.658-0.962)$ \\
\hline
\end{tabular}

${ }^{a}$ Odds ratios were adjusted for multiple birth, pre-gestational $\mathrm{BMI} \geq 28 \mathrm{~kg} /$ $\mathrm{m}^{2}$, pre-gestational hypertension, pre-gestational diabetes, placenta previa, preeclampsia and establishment of HDU

pregnancy and pre-gestational hypertension were not independent risk factors for ICU admission.

\section{Discussion}

This is the first study analyzing HDU in management of severe maternal morbidity in China. In this study from a large referral center in China, the ICU admission rate decreased by $20 \%$, whereas the severity of the cases increased after introduction of obstetric HDU. Nonetheless, the maternal mortality rate remained stable. Hypertensive disorders in pregnancy was the most common cause for ICU and HDU admission, while cardiac diseases and hemorrhage accounted for the second largest proportion in ICU and HDU cases respectively.

The research showed a rising trend of high-risk pregnancies in our population, which resulted in an increased demand for higher level of care. The rate of HDU admission in this study was $7.1 \%$, which was higher than the UK survey (4.2\%) [8] and lower than two single center reports from India (11.1 and $11.2 \%)$ [9, 10]. The variations were likely to be influenced by annual birth rate, characteristics of the population, and criteria for transferring women to ICU. Similar to the literatures [9, 10], our research showed the main indications for high dependency care were obstetric reasons and the introduction of HDU caused a significate reduction in obstetric hemorrhage and hypertensive disorders related ICU admission, while women with severe medical co-morbidities and women with organ dysfunction still needed ICU care. It is notable that our obstetric HDU has a relatively large capacity and situated in the obstetric wards rather than close to the ICU; therefore, high-risk patients who would otherwise have been managed in general ward were managed in HDU. For example, patients requiring intravenous antihypertensive drugs or close monitoring of input and output now received specialized care by the HDU team. Whether obstetric HDU can improve patient outcomes remained uncertain [11]. In our practices, doctors and nurses believed that the integration of intermediate care in obstetric practice benefited patient safety and reduced work stress.

ICU provides invasive monitoring and organ support to women with severe morbidity. Since we triaged women towards different level of units, the case severity of ICU admission increased significantly, as we showed a rising trend in the APACHE II score and length of stay among ICU cases. Consistent with other studies $[3,5,12]$, cardiac diseases and other medical disorders accounted for the majority of non-obstetric indications in our ICU admissions and often required invasive hemodynamic monitoring, mechanical ventilation and multidisciplinary management. We also noticed that women with non-obstetric indications were more complicated than those with obstetric indications. Eight out of nine maternal mortalities in our cohort were due to nonobstetric causes and the proportion of medical disorder indicated ICU admissions remained unchanged after HDU introduction.

In addition to triage of less severe patients to HDU, the decrease in obstetric hemorrhage related ICU admission was also attributable to our institutional quality improvement project targeted to prevent complications in severe postpartum hemorrhage [13]. After the introduction of a criteria-based clinical audit, maternal morbidity rate and blood transfusion rate in postpartum hemorrhage cases decreased significantly. This finding is in agreement with a multi-center research that instituting a protocol for the treatment of maternal hemorrhage significantly reduced the incidence of blood transfusion [14].

The present study showed a significant association between pre-eclampsia and ICU admission, which was similar with previous researches $[12,15]$. In Lin's study, obstetric ICU admission was nearly 4 times higher in women with hypertensive disorders in pregnancy [12]. Hitti et al. found that preeclampsia with severe features had the strongest association with severe maternal complications, followed by preeclampsia without severe features and chronic hypertension [15]. It is interesting that in the current study women with preeclampsia had over 7 folds higher risk for ICU admission, while chronic hypertension was not an independent risk factor. The reason for this disparity might be related to our referral system, where most women with chronic hypertension were referred to tertiary care in early pregnancy [16], whereas women with pre-eclampsia were often late-referrals with delayed diagnosis and suboptimal management [17]. This fact also implied that patients with uncomplicated hypertension could be safely managed in HDU, while 
preeclamptic patients with organ dysfunction frequently need intensive care. To reduce severe maternal complications related to preeclampsia, potential strategies include first trimester screening and prediction [18], low-dose aspirin prevention, early detection and tighter blood pressure control $[19,20]$.

\section{Generalizability and Limitations}

The findings in this paper are based on a tertiary care, university-based hospital that cares for a higher percentage of medically complicated pregnancies. The high medical acuity of this population lends itself to a close evaluation of the changes in the characteristics of ICU admissions after introduction of obstetric HDU. A major limitation of our study is that it relies on a single center data. The outcomes would be fairly comparable with referral centers and may not be representative to population of our region. Therefore, it is possible that the increased trend in medical co-morbidities this study is caused by an increased number of referral and not a reflection of trend in the population. Moreover, there is no standardized criteria for HDU admission, which hampers comparison of data between reports and the interpretation of outcomes.

\section{Conclusions}

Our study highlights the increasing role of HDU in management of severe maternal morbidities. Shifting of care for women with severe postpartum hemorrhage and hypertensive disorders in pregnancy to HDU may spare the ICU service for the more complex medical conditions. Whether providing intermediate care is cost-effective needs to be investigated in further studies.

\section{Abbreviations}

HDU: High dependency unit; ICU: Intensive care unit; OR: Odds ratios; Cl: Confidence intervals; aOR: adjusted odds ratios; BMI: Body mass index.

\section{Acknowledgment}

The authors are grateful for the assistance of healthcare staff in the department of ICU.

\section{Authors' contributions}

Study concept and design: NG. Acquisition of data: NG, YZ. Analysis and interpretation of data: NG. Drafting of the manuscript: NG; YD. All authors have read and approved the manuscript.

\section{Funding}

The present study was supported by Foundation for Critical Care in Maternal Medicine, Jiangsu Commission of Health, China.

\section{Availability of data and materials}

The datasets used and analyzed during the current study are available from the corresponding author on reasonable request.

\section{Declarations}

Ethics approval and consent to participate

The research has been approved by the Ethical Committee of Nanjing Drum Tower Hospital. A waiver of individual informed consent was granted by the Ethical Committee of Nanjing Drum Tower Hospital. All methods were carried out in accordance with relevant guidelines and regulations.

\section{Consent for publication}

Not required.

\section{Competing interests}

The authors have no conflicts of interest.

Received: 28 July 2021 Accepted: 15 February 2022

Published online: 21 February 2022

\section{References}

1. Committee on Practice Bulletins-Obstetrics. ACOG Practice Bulletin No. 211: Critical Care in Pregnancy. Obstet Gynecol. 2019;133:e303-19.

2. Liu J, Song L, Qiu J, Jing W, Wang L, Dai Y, et al. Reducing maternal mortality in China in the era of the two-child policy. BMJ Glob Health. 2020;5(2):e002157.

3. Zhao Z, Han S, Yao G, Li S, Li W, Zhao Y, et al. Pregnancy-Related ICU Admissions From 2008 to 2016 in China: A First Multicenter Report. Crit Care Med. 2018;46(10):e1002-9.

4. Ryan M, Hamilton V, Bowen M, McKenna P. The role of a high-dependency unit in a regional obstetric hospital. Anaesthesia. 2000;55:1155-8.

5. Liu Y, Tan G, Cheng S, Sun X. The ICU Is Becoming a Main Battlefield for Severe Maternal Rescue in China: An 8-Year Single-Center Clinical Experience. Crit Care Med. 2017:45:e1106-10.

6. Knaus WA, Draper EA, Wagner DP, Zimmerman JE. APACHE II: a severity of disease classification system. Crit Care Med. 1985:13(10):818-29.

7. Say L, Souza JP, Pattison RC. Maternal near miss - towards a standard tool for monitoring quality of maternal health care. Best Pract Res Clin Obstet Gynaecol. 2009;23:287-96.

8. Hussain S, Srinivas K, Yadthore S, Collis R. Obstetric high-dependency care: a 2005-06 UK survey of practice and facilities. Int J Obstet Anesth 2011;20(1):100-1.

9. Murki A, Dhope S, Kamineni V. Feto-maternal outcomes in obstetric patients with near miss morbidity: an audit of obstetric high dependency unit. J Matern-Fetal Neonatal Med. 2017;30(5):585-7.

10. Dattaray C, Mandal D, Shankar U, Bhattacharya P, Mandal S. Obstetric patients requiring high-dependency unit admission in a tertiary referral centre. Int J Crit IIIn Inj Sci. 2013;3(1):31-5.

11. Vincent $\mathrm{L} L$, Rubenfeld GD. Does intermediate care improve patient outcomes or reduce costs? Crit Care. 2015;19(1):89.

12. Lin L, Chen YH, Sun W, Gong JJ, Li P, Chen JJ, et al. Risk factors of obstetric admissions to the intensive care unit: An 8-year retrospective study. Medicine (Baltimore). 2019;98(11):e14835.

13. Zhou Y, Gu N, Xu X, Zhou H, Lu X, Wang Z, et al. Effect of continual quality audit in severe postpartum hemorrhage on its standardized diagnosis and treatment and the complications. Chinese J Pract Gynecol Obstetrics. 2021;37:1039-43 (Chinese).

14. Shields LE, Wiesner S, Fulton J, Pelletreau B. Comprehensive maternal hemorrhage protocols reduce the use of blood products and improve patient safety. Am J Obstet Gynecol. 2015;212(3):272-80.

15. Hitti J, Sienas L, Walker S, Benedetti TJ, Easterling T. Contribution of hypertension to severe maternal morbidity. Am J Obstet Gynecol. 2018;219(4):405. e1-7.

16. Zheng TQ, Yang H. Maternal-Fetal Medicine in China. Maternal-Fetal Medicine. 2020;2:48-55.

17. Gu N, Wang Z, Hu Y, Dai Y. Clinical audit of intensive care unit admission relating to hypertensive disorders of pregnancy. Chin J Perinat Med. 2018;21(9):585-91 (Chinese).

18. Chaemsaithong P, Sahota DS, Poon LC. First trimester preeclampsia screening and prediction. Am J Obstet Gynecol. 2020;S0002-9378(20):30741-9. 
19. Duhig KE, Myers J, Seed PT, Sparkes J, Lowe J, Hunter RM, et al. Placental growth factor testing to assess women with suspected pre-eclampsia: a multicentre, pragmatic, stepped-wedge cluster-randomised controlled trial. Lancet. 2019;393:1807-18.

20. Magee LA, von Dadelszen P, Singer J, Lee T, Rey E, Ross S, et al. The CHIPS randomized controlled trial (control of hypertension in pregnancy study): is severe hypertension just an elevated blood pressure? Hypertension. 2016;68:1153-9.

\section{Publisher's Note}

Springer Nature remains neutral with regard to jurisdictional claims in published maps and institutional affiliations.

- fast, convenient online submission

- thorough peer review by experienced researchers in your field

- rapid publication on acceptance

- support for research data, including large and complex data types

- gold Open Access which fosters wider collaboration and increased citations

- maximum visibility for your research: over $100 \mathrm{M}$ website views per year

At BMC, research is always in progress.

Learn more biomedcentral.com/submissions 